\title{
PERCEPCIÓN DE ESTUDIANTES DEL I CICLO DE ENSEÑANZA GENERAL BÁSICA ACERCA DE LA VIOLENCIA ESCOLAR PERCEPTION OF STUDENTS OF I CYCLE OF BASIC GENERAL EDUCATION ABOUT SCHOOL VIOLENCE
}

\author{
Volumen 14, Número 1 \\ Enero - Abril \\ pp. 1-23
}

Este número se publicó el 30 de enero de 2014

Jenny María Artavia Granados

Revista indizada en REDALYC, $\underline{\text { SCIELO }}$

Revista distribuida en las bases de datos:

CATÁLOGO DE LATINDEX, IRESIE, CLASE, DIALNET, DOAJ, E-REVIST@S, SHERPA/ROMEO, QUALIS, MIAR

Revista registrada en los directorios:

ULRICH'S, REDIE, RINACE, OEI, MAESTROTECA, PREAL, $\underline{\text { CLASCO }}$ 


\title{
PERCEPCIÓN DE ESTUDIANTES DEL I CICLO DE ENSEÑANZA GENERAL BÁSICA ACERCA DE LA VIOLENCIA ESCOLAR PERCEPTION OF STUDENTS OF I CYCLE OF BASIC GENERAL EDUCATION ABOUT SCHOOL VIOLENCE
}

\begin{abstract}
Jenny María Artavia Granados ${ }^{1}$
Resumen: El presente artículo expone el grado de percepción de la violencia que poseen las niñas y los niños del I Ciclo de la Enseñanza General Básica de una escuela de la Dirección Regional de Educación de Occidente, Costa Rica, a partir del análisis de los comportamientos cotidianos que se presentan en los juegos e interacciones sociales que se desarrollan durante los recreos. Este es producto de una investigación de tipo mixta, que como técnica de recolección de datos empleó un cuestionario. El grupo de estudiantes elegido para el estudio estuvo conformado por doscientos ochenta y dos estudiantes de I, II y III grado. Algunas de las conclusiones indicó un alto porcentaje de los estudiantes identifican la existencia de violencia en acciones como empujar, golpear, dar zancadillas, entre otras. Asimismo, un alto porcentaje también manifestó que sí hay violencia cuando otros los intimidan o acosan. Respecto a la violencia psicológica, un alto porcentaje logró percibir su existencia en las acciones que se les presentaron en los respectivos ítems. También, señalaron que sí existe violencia cuando son insultados, les dicen apodos y usan palabras soeces. Además, un alto porcentaje logró reconocer la existencia de la violencia sexual. Sin embargo, las y los estudiantes de II grado y III grado consideran que no existe violencia en acciones como cuando no los dejan jugar, les quitan la merienda o los útiles escolares.
\end{abstract}

Palabras clave: VIOLENCIA ESCOLAR, PERCEPCIÓN, NIÑO ESCOLAR, EDUCACIÓN PRIMARIA, COSTA RICA

\begin{abstract}
This article exposes the degree of perception of violence that children of the I cycle of the Basic General Education have, of one school in the Western Regional Bureau of Education, Costa Rica, based on the analysis of the everyday behaviors that occur in games and social interactions that take place during the breaks. This article is a product of a mixed type research, in which one of the data collection techniques employed was a questionnaire. The group of students selected for the study consists of two hundred and eighty-two students of I, II and III grade. Some of the findings show that: this group of students, in a high percentage identified the existence of violence in actions such as: pushing, hitting, and tripping, among others. In addition, a high percentage of them also indicates that there is violence when others intimidate them or harass them. About the psychological violence, a high percentage perceives its existence, in actions that were presented in the respective items. However, students of II grade and III grade consider that there is no violence in actions such as: when they are not allowed to play or someone takes their snack or school supplies. Also students point out that there is violence when they are insulted; nicknames are given to them and the use of swear or bad words. In addition a high percentage manages to identify the existence of sexual violence.
\end{abstract}

Key words: SCHOOL VIOLENCE, PERCEPTION, SCHOOL CHILD, PRIMARY EDUCATION, COSTA RICA

\footnotetext{
${ }^{1}$ Docente del Departamento Ciencias de la Educación en la Sede de Occidente de la Universidad de Costa Rica. Doctora en Educación de la Universidad Estatal a Distancia, Máster en Psicopedagogía de la Universidad Estatal a Distancia, Licenciada en Educación Primaria y en Administración Educativa de la Universidad de Costa Rica.
}

Dirección electrónica: jargra@gmail.com

Artículo recibido: 10 de junio, 2013

Devuelto para corrección: 4 de noviembre, 2013

Aprobado: 12 de diciembre, 2013 


\section{Introducción}

El fenómeno de la violencia ha invadido la vida cotidiana de las personas, se ha infiltrado en barrios, calles, talleres, oficinas, hogares y ha llegado también a las instituciones educativas. Los medios de comunicación se han encargado de mostrar a la sociedad los niveles de violencia generados en escuelas, colegios y universidades, tanto a nivel nacional como internacional. A gran cantidad de niños y jóvenes les ha correspondido ser testigos de violencia física o psicológica, maltrato o intimidación verbal.

La violencia física deja huellas en el cuerpo del niño o joven. Es un tipo de violencia fácil de percibir: golpes, empujones, zancadillas, pellizcos, entre otros. Según Álvarez, Álvarez, González, Núñez y González (2006), "la violencia física es aquella en la que existe un contacto material para producir el daño y se puede distinguir entre violencia directa e indirecta" (p. 687). En la violencia directa, el agresor actúa de forma inmediata sobre su víctima golpeándola, mientras que en la violencia indirecta, el agresor actúa sobre un objeto o material (robo o destrucción) que le pertenece a la víctima.

Otro tipo de violencia es aquella denominada psicológica, la cual, como bien lo indica Arias (2009), "se manifiesta por medio de agresiones verbales, intimidaciones, maltrato, amenazas, marginaciones, u otras" (p. 45). Es un tipo de violencia que se manifiesta de forma oculta, solapada, pero que causa mucho daño psicológico a la víctima.

Respecto al maltrato entre iguales, este también se lleva a cabo de forma oculta. La víctima sufre en silencio la intimidación o el acoso proveniente de su victimario. Para Cerezo (2002) la intimidación causa en la víctima daño moral, físico y tensión nerviosa. La víctima siente una constante amenaza y no sabe cómo salir de esta situación, lo que le provoca miedo.

La violencia verbal es el resultado de un lenguaje soez que insulta, degrada y humilla a la víctima. Según Cabrera (2005), este tipo de violencia tiende a ser menos perceptible, pues no se visualiza ningún acto físico violento contra otro, pero sí se ejerce violencia a través del lenguaje. A través de la violencia verbal se expresan insultos, apodos, humillaciones, burlas y otros. Muchas de las expresiones que se dejan escuchar a partir de la violencia verbal, están relacionadas con rasgos físicos sobresalientes de la persona, con características psicológicas, con el color de la piel o con la clase social. 


\section{Percepción del fenómeno de la violencia que poseen el niño y la niña en edad escolar}

Las niñas y los niños se desarrollan en diferentes ambientes, como el familiar, escolar, comunal, entre otros, y en cada uno de estos podrían ser testigos, víctimas o victimarios de diversos comportamientos de violencia. El fenómeno de la violencia no escapa a la vivencia cotidiana de niños, niñas o jóvenes. Estos tienen que enfrentar y convivir con diferentes tipos de violencia: abierta o manifiesta y estructural.

Respecto a la violencia estructural, esta se desarrolla en las instituciones culturales, económicas y sociales, y se expresa mediante la discriminación y exclusión de sectores de la población según la edad, género, nivel social, económico, etnias, entre otros. Para Abad (2002) "somos parte de una sociedad con una descomunal asimetría de poder (sostenida por la fuerza), especialmente de decisión sobre la distribución de los recursos, bautizado en los 60 ' por Galtung como violencia estructural o injusticia social" (p. 35). Este tipo de violencia no es percibida como tal por la mayoría de la población, porque el Estado se encarga justamente de regular y administrar las conductas de los colectivos de la sociedad a través de valores, costumbres, folclore, normas, leyes, ritos, entre otros. La población tiende a sentir los efectos de este tipo de violencia cuando la discriminación y la exclusión niegan a grandes estratos de la colectividad aspectos fundamentales que tienen que ver con género, ciudadanía, política y derecho (tener techo digno, alimento, vestido, trabajo, educación, entre otros). La violencia, desde el punto de vista económico, se hace visible cuando existen problemas relacionados con la pobreza, el desempleo, la explotación, el robo, la corrupción y el deterioro de servicios básicos como son la salud, vivienda y educación. A partir de la violencia económica, surge la violencia social, y de esta se desprende la violencia intrafamiliar, la violencia escolar, la violencia sexual, la violencia que transmiten los medios de comunicación y la violencia comunal, entre otras. Palomero y Fernández (2001) manifiestan que,

Esta violencia estructural, que se halla incorporada al sistema y que se manifiesta de múltiples formas y en especial a través de todo tipo de injusticias y desigualdades sociales, económicas, jurídicas, de género, raciales o de cualquier índole, afecta especialmente a los más débiles y causa daño a todos los seres humanos en general, pues es la responsable de que el nivel de realización real de las personas sea inferior a su nivel de realización potencial. (p. 26) 
Por tanto, la violencia estructural se logra percibir como tal, cuando las personas ven afectados aspectos que tienen que ver con su modus vivendi, su autorrealización, derechos, seguridad ciudadana, desarrollo humano, entre otros. Los niños y jóvenes que viven dentro de la dinámica de la violencia estructural, tienden a reproducir la violencia que los victimiza, y lo hacen justamente a través de la violencia manifiesta.

La violencia abierta o manifiesta, por su parte, se ejerce directamente sobre el cuerpo. Los efectos de este tipo de violencia son totalmente visibles: golpes, luchas, forcejeos, disparos, y también se producen actos de vandalismo, en los cuales se causa daño o destrucción de objetos de la persona víctima de este tipo de violencia. La violencia manifiesta puede ser más fácilmente percibida por niños, jóvenes o adultos, porque el impacto lo recibe la víctima de forma inmediata a través de violencia física, violencia verbal, acoso, intimidación y violencia psicológica. En la escuela, el niño y la niña perciben este tipo de manifestaciones de violencia, a partir de las interrelaciones que se suscitan entre sus compañeras y compañeros durante los procesos educativos que se desarrollan en los diferentes espacios educativos como el salón de clases y también en los espacios de recreación que se llevan a cabo en el recreo escolar.

Las manifestaciones de violencia que se originan entre estudiantes durante el tiempo lectivo, como ya se hizo referencia anteriormente, se producen de forma explícita o directa a través de la violencia física o verbal, o se suscita de forma oculta a través de la violencia psicológica, las intimidaciones, el maltrato, las amenazas y las marginaciones.

\section{Metodología}

Este artículo surge como producto de una investigación de índole mixta realizada por Artavia (2012), en la que se analiza si los juegos y las interrelaciones sociales que se desarrollan durante los recreos entre las y los estudiantes de I Ciclo de la Enseñanza General Básica de una escuela de la Región Educativa de Occidente, tienen relación con los comportamientos de violencia en esta escuela. Uno de los objetivos específicos de la investigación fue identificar el grado de percepción de la violencia que poseen los niños, a partir de los juegos e interacciones sociales que se desarrollan en los recreos. Como parte de este estudio, se procedió a aplicar un cuestionario a los niños y niñas de I, II y III grado de dicha escuela. Este artículo se generó a partir de los datos que aportaron dos de los ítemes 
del cuestionario aplicado, los cuales procuraron indagar si las y los estudiantes identificaban en las acciones que se les presentaron comportamientos violentos.

La población estudiantil sujeto del estudio, estuvo conformada por 282 estudiantes cuyas edades oscilaron entre los 6 y 11 años. La población está distribuida en tres grados: I grado, 97 niños (57 niños y 40 niñas); II grado, 99 niños (49 niños y 50 niñas); y III grado, 86 niños (36 niños y 50 niñas).

\section{¿Cuál es la percepción que tienen niñas y niños en edad escolar acerca del fenómeno de la violencia?}

Como parte del cuestionario que se pasó a las y los estudiantes, se les presentó una serie de dibujos donde se podía observar niños y niñas que realizaban acciones tanto violentas como no violentas. El objetivo de este ítem era precisamente obtener información que permitiera a la investigadora reconocer la percepción que tienen niños y niñas del I Ciclo de la escuela en estudio respecto a los comportamientos violentos y no violentos. En primera instancia, se presentan cinco dibujos donde los personajes realizan una serie de actividades que no son violentas: jugar canicas, jugar con una manguera, jugar con máscaras, jugar con un barrilete o cometa y, por último, jugar a hacerse cosquillas.

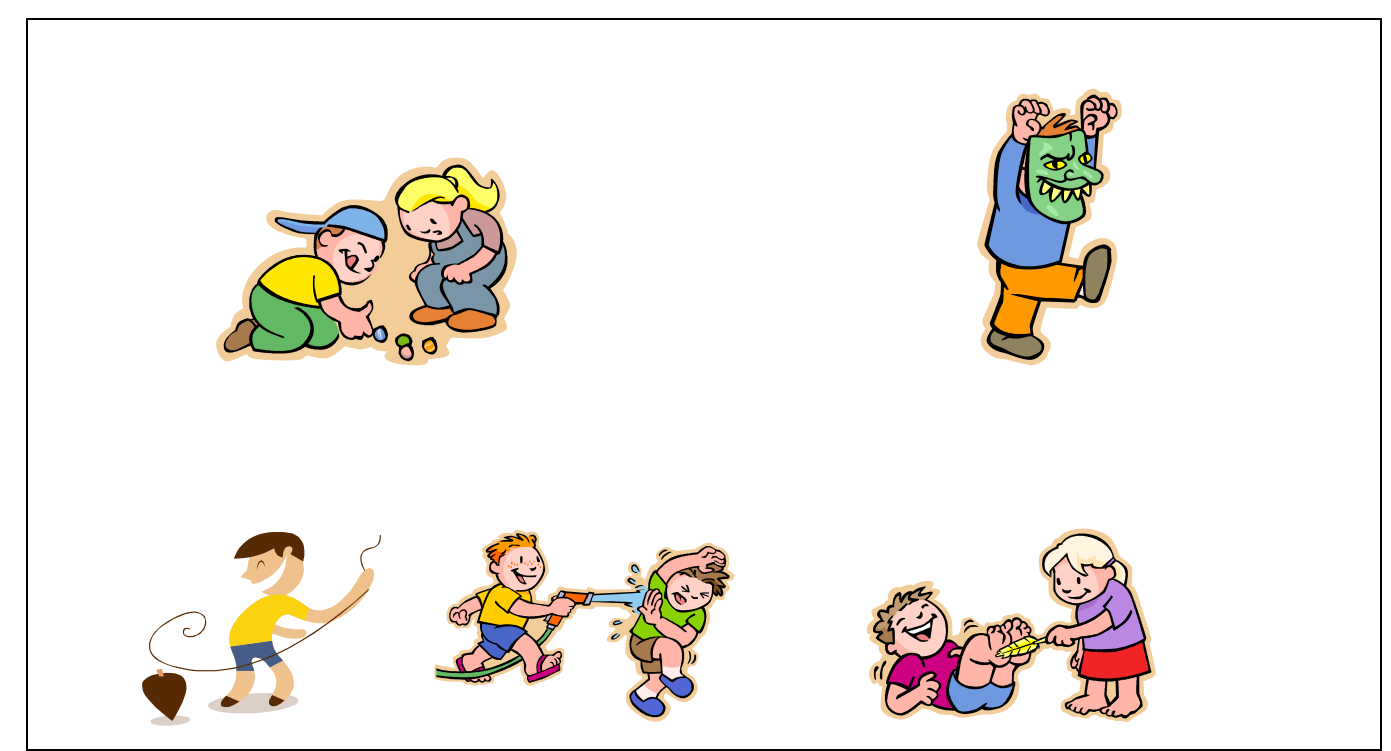

Ilustración 1: llustración de juegos y travesuras que realizan niños y niñas 
Como resultado de la selección realizada por las y los estudiantes, una considerable cantidad de niños y niñas de los tres grados encontraron que en dos de los dibujos que se les presentó, existían acciones violentas entre los niños de la ilustración 1. Uno de los dibujos presenta a un niño mojando con una manguera a otro. En el siguiente dibujo, un niño que lleva puesta una máscara, y trata de asustar. Ambos dibujos fueron elegidos porque se consideró que no representaban acciones de violencia entre sus personajes, sino que, por el contrario, ilustran momentos de juego o travesura entre niños. Sin embargo, las y los estudiantes sujetos del estudio sí consideraron que los personajes de los dibujos llevaban a cabo acciones violentas.

En el dibujo donde un niño moja con una manguera a otro, 69 estudiantes de I grado (71\%), 54 de II (55.5\%) y 51 de III grado 59\%) señalan que en esta ilustración sí hay violencia. Respecto al dibujo que ilustra a un niño que juega con una máscara, un alto número de estudiantes de los tres grados escolares consideraron que sí hay comportamiento violento en la acción del niño del dibujo. Inclusive, resulta importante señalar que tres estudiantes indicaron que en el dibujo donde una niña hace cosquillas a un niño, existe un comportamiento violento.

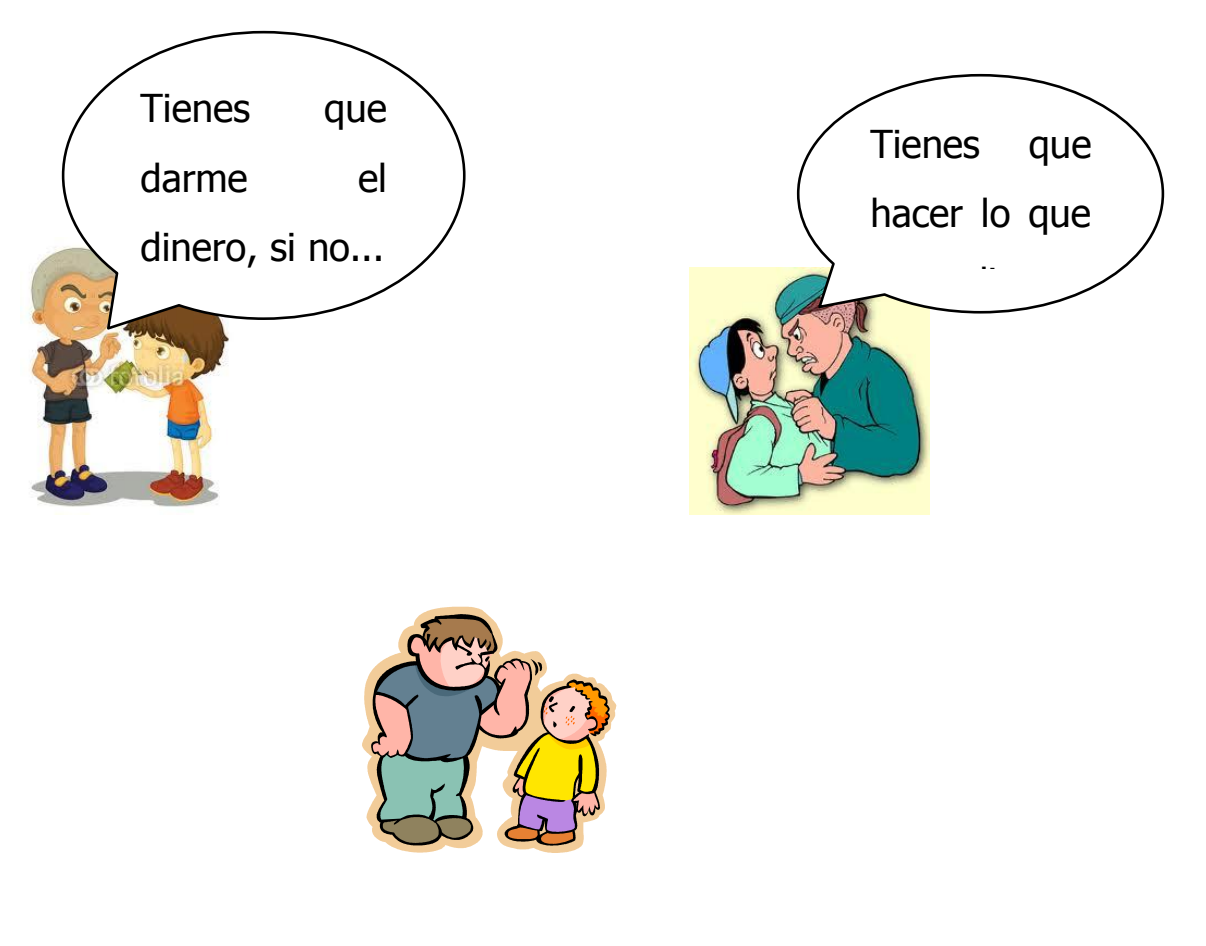

Ilustración 2: Ilustración de niños que amenazan a otros 
Se presentaron también tres dibujos donde se pueden observar a niños que están siendo expuestos a amenazas por parte de otros chicos (Ilustración 2). Como resultado de la opinión de las y los estudiantes sujetos del estudio, un alto porcentaje de la población total de los tres grados (95\%), consideraron que en estas ilustraciones sí existen comportamientos violentos entre niños. En el dibujo que ilustra a dos niños en un acto donde uno de ellos entrega su dinero a otro, porque, tal y como lo indica la figura que representa la expresión del victimario sobre la víctima, este le señala "Tienes que darme el dinero, si no...", 88 estudiantes de I grado (91\%) visualizan que en esta acción existe un comportamiento violento, así como 85 estudiantes de II grado (86\%) y, 76 de III grado (88\%), quienes señalan también la presencia de un acto violento en esta relación interpersonal entre niños. Resulta importante indicar que un menor número de III grado (35 niños) no percibe que en este acto se esté dando una acción violenta. Arellano, Chirinos, López y Sánchez (2007) observan que este tipo de maltrato es de tipo económico porque

Se busca el control de los recursos económicos de la víctima, esto implica una destrucción o privación del sustento y/o de su propiedad, se manifiesta cuando al niño o al joven se le sustrae o se le exige el dinero que lleva a la escuela, cuando le roban sus pertenencias, o se esconde o destruyen sus posesiones. (p. 7)

Ahora bien, respecto al dibujo donde hay un niño de mayor edad (victimario) que ostenta sobre otro de menor edad (víctima) un comportamiento donde toma a la víctima de la camisa, en señal de provocación y advertencia, y le dice "Tienes que hacer lo que yo te diga", un alto número de niños y niñas de I, II y III grado señalan que en esta relación se da un comportamiento violento.

Por último, esta ilustración muestra un dibujo donde un niño mayor (victimario) hace una señal con su brazo donde le indica a otro niño menor que le va a pegar. Al respecto, al igual que en los otros dos dibujos, un alto porcentaje de las y los estudiantes encuestados (90\%), consideran que sí hay violencia. Este tipo de gestos agresivos y groseros son los que ostentan los victimarios que suelen ser fuertes físicamente, y a veces mayores, sobre víctimas que generalmente suele verse débil físicamente y menores que el agresor. Este tipo de desequilibrio es una conducta que permite que se dé maltrato entre iguales. Al respecto, Hernández (2008) manifiesta que "las conductas agresivas dentro de la escuela no se 
reducen a acontecimientos de violencia física, sino que se trata de abusos de poder por parte de personas más fuertes en contra de otra o de otras más débiles" (p. 4).

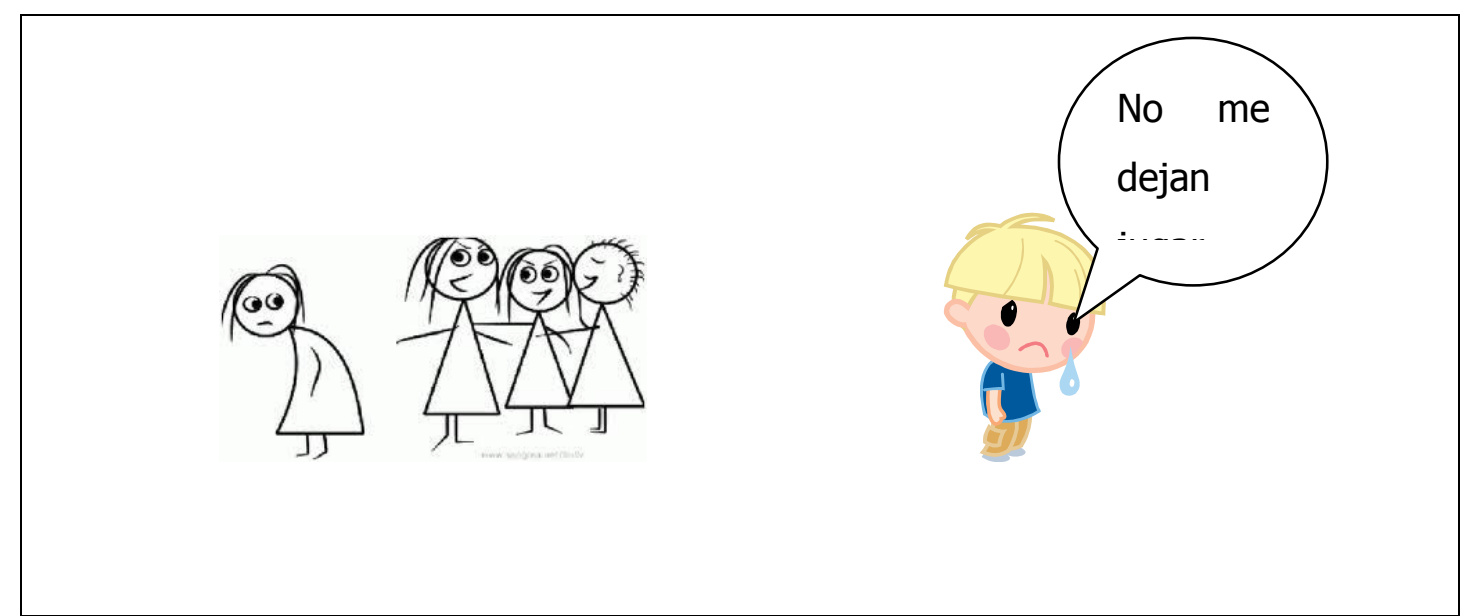

Ilustración 3: Ilustración de niños y niñas que sufren violencia psicológica

Como parte de la violencia psicológica que ejercen los victimarios sobre las víctimas, se muestra un comportamiento violento que incluye estrategias que procuran ignorar, excluir y aislar a la víctima del resto de sus compañeros y compañeras. En la ilustración 3 se presentan casos donde las y los estudiantes sujetos del estudio debían valorar si hay o no acciones violentas, específicamente aquellas que tienen que ver con comportamientos de rechazo y exclusión. En cuanto a la percepción de las y los niños respecto a ambos dibujos, un alto número de estos consideran que sí existen comportamientos violentos en el dibujo que ilustra a un grupo de niñas que conversan entre ellas, quizá haciendo algún comentario negativo respecto de otra que se marcha del grupo muy triste, porque al parecer no es aceptada por sus compañeras. Benítez y Justicia (2006) manifiestan que "algunos datos apuntan que aunque los niños tienden a practicar con más frecuencia malos tratos físicos y verbales directos, ambos sexos aparecen igualados en cuanto a los maltratos indirectos, tal como la exclusión social" (p. 159).

Respecto a las y los estudiantes de I grado, 83 (85,5\%) opinan que sí existe violencia en esta acción. Por otra parte, de la población estudiantil de II y III grado, 73 estudiantes (74\%) y 65 estudiantes (75,5\%), respectivamente, también consideran que existe en este tipo de relación, un comportamiento violento. 
Con respecto al dibujo donde un niño llora y exclama "No me dejan jugar", las y los niños encuestados consideran que sí existe violencia. Sin embargo, en comparación con el dibujo anterior, este no logra obtener un alto número de puntuación a favor entre los varones de II y III grado. Los de I grado un 65\% (37 niños) sí indica que existe un comportamiento violento. Respecto a los niveles de II y III grado, son las niñas, en un alto porcentaje, las que consideran que en la acción que se presenta en la ilustración 3 sí existe un comportamiento violento. Pareciera que los varones de II y III grado no perciben que en la acción donde alguien no les permite jugar, exista realmente una acción violenta.

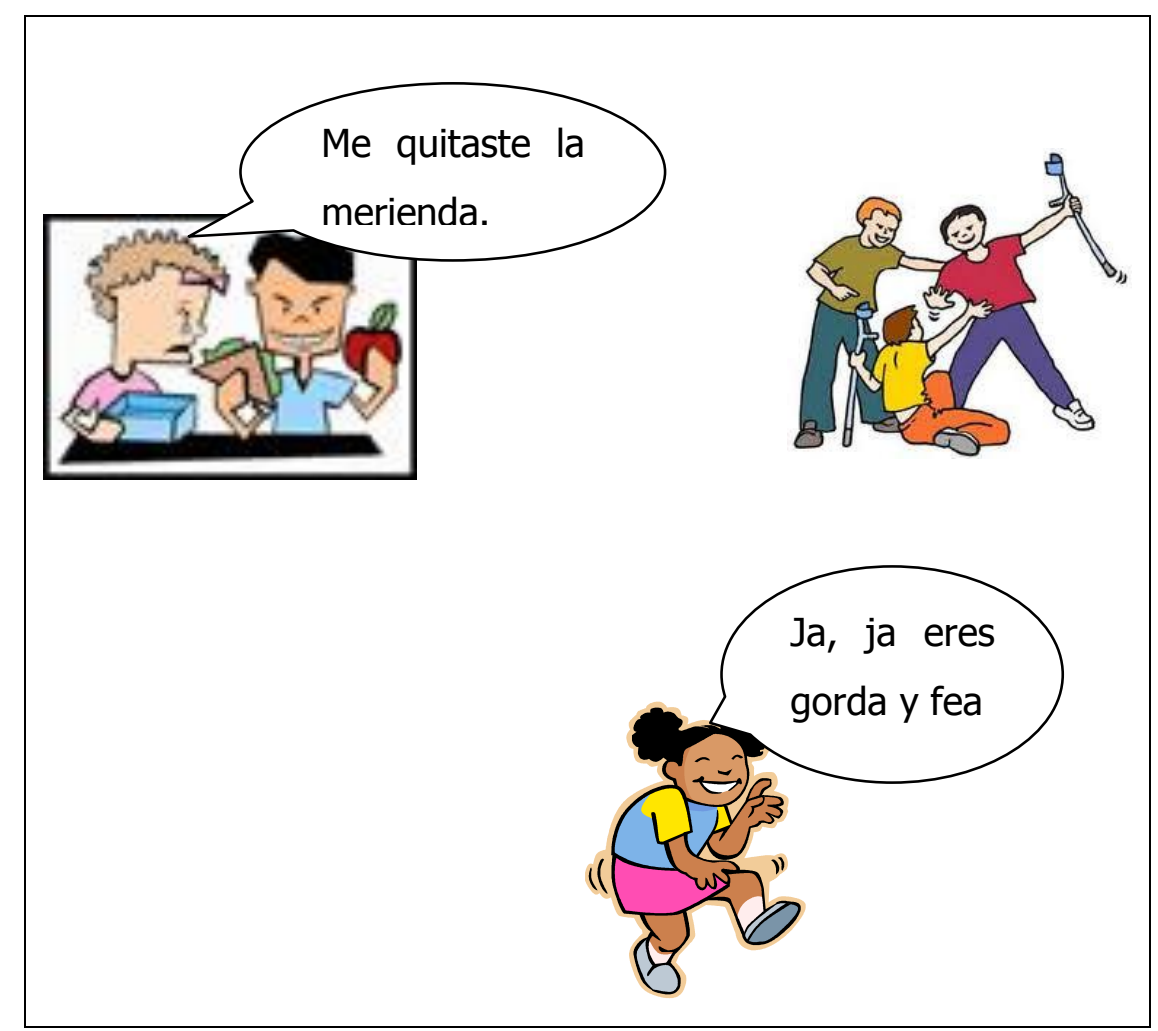

Ilustración 4: llustración comportamientos violentos de índole psicológica.

La ilustración 4 muestra tres dibujos que se le presentó a la población en estudio, y que ilustran comportamientos violentos de índole psicológica. A las y los estudiantes se les presentaron tres dibujos donde víctimas y victimarios realizan acciones que tal vez no se evidencian tan fácilmente, pero que podrían provocar daños menos visibles de tipo psicológico o emocional. 
El primer dibujo que se muestra, hace referencia a un niño (victimario) que le quita a otro su merienda, mientras que este (víctima) llora, y sólo acata a decirle: "Me quitaste la merienda". Ante esta ilustración, un alto número de estudiantes de los tres grados consideraron que esta acción era un comportamiento violento. Los resultados obtenidos permiten identificar que tanto niños como niñas coinciden en que, ante el hecho de que alguien le quite a otro la merienda, existe un acto de violencia. Al respecto se encontró que 83 de los estudiantes de I grado (85,5\%), 75 de II grado (76\%) y 75 de III grado (87\%) opinan que en esta acción existe un comportamiento violento.

En cuanto a la segunda ilustración, esta presenta a un niño que posee una minusvalía. Para caminar requiere de sus bastones. Un grupo de niños (victimarios) se aprovecha de su indefensión para quitarle uno de sus bastones y hacerlo luchar para que se lo devuelvan. Los victimarios se ríen de la situación en que han dejado a la víctima. Ante este dibujo, un alto número de niños de los tres grados consideraron que se está dando un acto de violencia.

El último dibujo de esta figura ilustra cómo una niña se ríe ofensivamente de otra, porque la víctima tiene ciertas características físicas. La frase que caracteriza la burla de esta niña es la siguiente: “Ja, ja, eres gorda y fea”. En su gran mayoría, las y los estudiantes señalaron en sus respuestas que sí existía un comportamiento violento. Las respuestas de los estudiantes de I grado permiten identificar que un 84,5\% de ellos percibe esta acción como violencia. Asimismo, un $80 \%$ de niños y niñas de II grado y $67 \%$ de III grado también logran identificar violencia en esta acción.

Resulta importante resaltar que las figuras 2, 3 y 4 representan formas en las que se puede ejercer conductas de violencia entre estudiantes. En ellas el énfasis se centra en tipos de violencia que se manifiesta de forma oculta, tal es el caso de la violencia de índole psicológica y de exclusión social, las cuales podrían pasar desapercibidas. Sin embargo, de acuerdo con los resultados obtenidos, se puede indicar que la población estudiantil de la escuela en estudio logra percibir la existencia de estos tipos de violencia. 


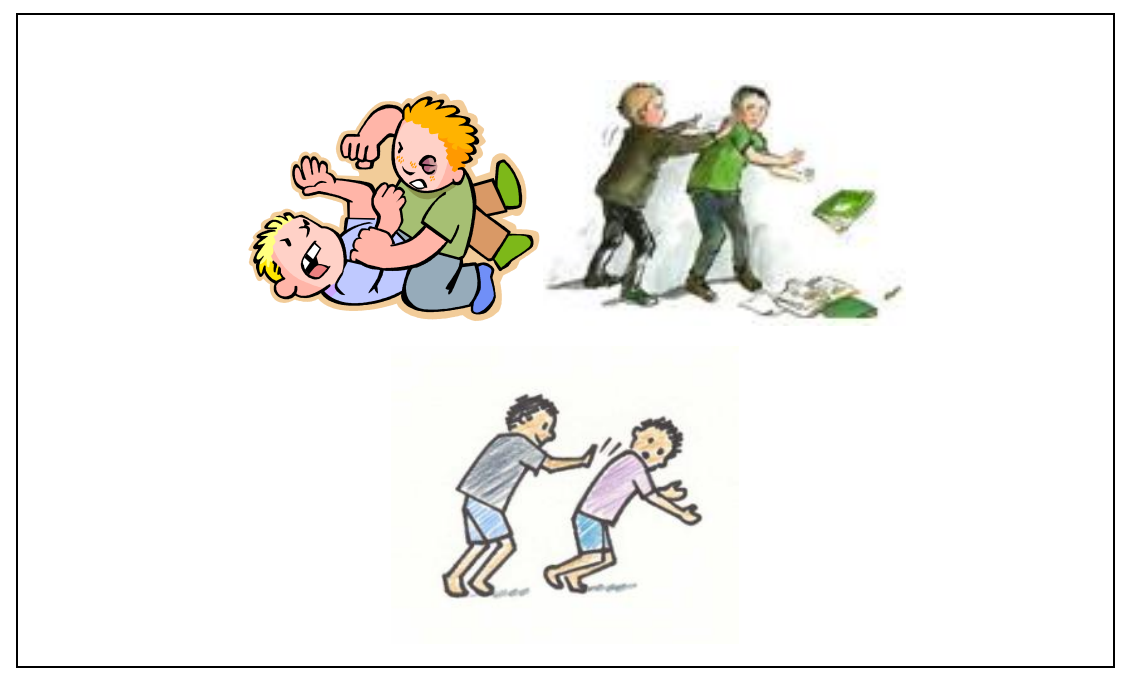

Ilustración 5: Ilustración de comportamientos de violencia física.

La ilustración 5 muestra los dibujos que se presentaron en el cuestionario a la población en estudio, relacionados con comportamientos violentos de tipo físico. A las y los estudiantes se les mostraron tres dibujos en los que se dejan ver niños golpeándose o empujando a otro compañero. Los resultados hallados reflejan que una gran mayoría de niños y niñas manifiesta que en los tres dibujos se suscitan comportamientos violentos. En el análisis de cada uno de estos dibujos se encontró que, en el primer dibujo donde aparecen dos niños peleando, dándose golpes por la cara y otras partes del cuerpo, un alto número de niñas y niños de I y II grado (92\%) señala que sí existe una acción de violencia.

En el segundo dibujo que se presentó, se muestra a un niño que empuja a otro y hace que todos sus útiles escolares (cuadernos y libros) caigan al suelo. En el rostro del niño que recibe la acción violenta, se observa angustia y miedo por la acción de que fue víctima. Como respuesta de las y los estudiantes del estudio, un $89 \%$ logra identificar que este comportamiento es violento.

En cuanto al tercer dibujo, este muestra a un niño que empuja a otro con la aparente intención de hacer que caiga al suelo. Este tipo de comportamientos, tal y como lo señala la Fundación Piquer (2010), son típicos del acoso físico que sufren muchos niños y que se revela a través de empujones, patadas, zancadillas, golpes, entre otros. Estos son más frecuentes en la etapa de Primaria que en Secundaria. Al igual que en las acciones anteriores, un $85 \%$ de las y los niños consideran que en este tipo de comportamiento sí existe violencia. 
Como parte de los ítemes que conforman el cuestionario, se presentó también información relacionada con los comportamientos violentos que han vivido o podrían experimentar las y los estudiantes del estudio. A esta población se le mostró un listado de treinta situaciones que podrían sucederles a ellos y ellas durante el desarrollo del recreo, para que identificaran en cuál o cuáles de estas situaciones consideraban existían comportamientos violentos. Es importante señalar que la forma en que se pasó los cuestionarios a las y los estudiantes, permitió a la investigadora estar en contacto directo con la población, pues en el caso de las y los estudiantes de I grado, se trabajó en subgrupos de 5 estudiantes y se procedió a la lectura de cada uno de los ítemes del cuestionario. Esto permitió a la investigadora observar sus reacciones y escuchar los comentarios que realizaban respecto a lo que se les preguntaba. Con las y los niños de II y III grado también se tuvo la oportunidad de estar presente en los salones de clase de cada grupo. Se procedió a ir leyendo y guiando la resolución del cuestionario. Esto también permitió aclarar dudas, escuchar comentarios y observar sus reacciones ante la lectura de lo que se les cuestionaba.

Para lograr una mejor apreciación de los resultados de esta información, se organizaron los datos de acuerdo con los tipos de violencia: física, psicológica, verbal y de acoso sexual. Asimismo, se presentaron dos cuadros (números 6 y 7) con acciones donde no existen comportamientos violentos.

Desde la perspectiva de la violencia física, se presentaron tres situaciones o acciones que podrían suceder durante el transcurso del recreo. Estas se leen en el Cuadro 1.

\section{Cuadro 1}

San Ramón: Opinión de las y los estudiantes de I Ciclo Enseñanza General Básica acerca de las manifestaciones de violencia física que se podrían suscitar en los recreos, 2012

\begin{tabular}{|c|c|c|c|c|c|c|}
\hline \multirow{3}{*}{$\begin{array}{l}\text { Manifestaciones de violencia física } \\
\text { Si vienes corriendo y un niño te pega } \\
\text { una zancadilla. }\end{array}$} & \multicolumn{3}{|c|}{ Hay violencia } & \multicolumn{3}{|c|}{ No hay violencia } \\
\hline & \multicolumn{3}{|c|}{ I grado II grado III grado } & \multicolumn{3}{|c|}{ I grado II grado III grado } \\
\hline & $\begin{array}{c}91 \\
94,8\end{array}$ & $\begin{array}{r}90 \\
90,0\end{array}$ & $\begin{array}{l}81 \\
94,2\end{array}$ & $\begin{array}{l}5 \\
5,2\end{array}$ & $\begin{array}{l}10 \\
10,0\end{array}$ & $\begin{array}{c}5 \\
5,8\end{array}$ \\
\hline $\begin{array}{l}\text { Estás jugando y de pronto un niño(a) te } \\
\text { empuja. }\end{array}$ & $\begin{array}{r}88 \\
91,7\end{array}$ & $\begin{array}{r}90 \\
90,0 \\
\end{array}$ & $\begin{array}{r}85 \\
98,8 \\
\end{array}$ & $\begin{array}{c}8 \\
8,3 \\
\end{array}$ & $\begin{array}{l}10 \\
10,0\end{array}$ & $\begin{array}{c}1 \\
1,2 \\
\end{array}$ \\
\hline $\begin{array}{l}\text { Estás jugando con tus compañeros(as) } \\
\text { y de pronto uno de ellos comienza a } \\
\text { golpearte. }\end{array}$ & $\begin{array}{c}92 \\
95,8\end{array}$ & $\begin{array}{c}95 \\
95,0\end{array}$ & $\begin{array}{c}83 \\
96,5\end{array}$ & $\begin{array}{c}4 \\
4,2\end{array}$ & $\begin{array}{r}5 \\
5,0\end{array}$ & $\begin{array}{r}3 \\
3,5 \\
\end{array}$ \\
\hline
\end{tabular}

Fuente: Creación propia a partir del cuestionario aplicado a las y los estudiantes de los grupos en estudio, 2012. 
En relación con las acciones que conllevan violencia física, un alto porcentaje de las ni\{as y los niños de I, II y III grado consideran que en estos tres tipos de situaciones existen comportamientos violentos. Los empujones, zancadillas, golpes, entre otros, son comportamientos violentos que resultan ser fáciles de percibir como tales. Por tanto, se estima que cuando se suscitan entre estudiantes este tipo de comportamientos, el hecho queda totalmente expuesto ante los otros, llámense adultos o compañeros y compañeras. Asimismo, el niño o la niña perciben de forma rápida el maltrato físico de que son víctimas.

Cuadro 2

San Ramón: Opinión de las y los estudiantes de I Ciclo Enseñanza General Básica acerca de las manifestaciones de violencia psicológica que se podrían suscitar en los recreos, 2012

\begin{tabular}{|c|c|c|c|c|c|}
\hline \multirow{3}{*}{$\begin{array}{l}\begin{array}{l}\text { Manifestaciones de violencia } \\
\text { psicológica }\end{array} \\
\begin{array}{l}\text { Algunos compañeros (as) no te dejan } \\
\text { compartir con ellos algún juego o } \\
\text { conversación en el recreo. }\end{array}\end{array}$} & \multicolumn{2}{|c|}{ Hay violencia } & \multicolumn{3}{|c|}{ No hay violencia } \\
\hline & \multicolumn{2}{|c|}{ I grado II grado III grado } & \multicolumn{3}{|c|}{ I grado II grado III grado } \\
\hline & $\begin{array}{ll}78 & 64 \\
81,3 & 64,0\end{array}$ & $\begin{array}{c}47 \\
54,7\end{array}$ & $\begin{array}{l}18 \\
18,8\end{array}$ & $\begin{array}{l}36 \\
36,0\end{array}$ & $\begin{array}{l}39 \\
45,3\end{array}$ \\
\hline $\begin{array}{l}\text { Cuando alguien te quita la merienda o } \\
\text { alguno de los útiles escolares (lápiz, } \\
\text { borrador, otros). }\end{array}$ & $\begin{array}{lc}86 & 79 \\
89,6 & 79, \\
\end{array}$ & $\begin{array}{l}79 \\
91,9 \\
\end{array}$ & $\begin{array}{c}10 \\
10,4\end{array}$ & $\begin{array}{l}21 \\
21,0\end{array}$ & $\begin{array}{c}7 \\
8,1\end{array}$ \\
\hline $\begin{array}{l}\text { Cuando algunos compañeros (as) se } \\
\text { apartan de ti y no les gusta que } \\
\text { juegues o salgas con ellos al recreo. }\end{array}$ & $\begin{array}{cc}81 & 84 \\
84,4 & 84,0\end{array}$ & $\begin{array}{l}68 \\
79,1\end{array}$ & $\begin{array}{c}15 \\
15,6\end{array}$ & $\begin{array}{c}16 \\
16,0\end{array}$ & $\begin{array}{l}18 \\
20,9\end{array}$ \\
\hline
\end{tabular}

Fuente: Creación propia a partir del cuestionario aplicado a las y los estudiantes de los grupos en estudio, 2012.

El cuadro 2 agrupa una serie de acciones que pueden suceder durante el desarrollo de los recreos. Este tipo de comportamientos violentos se suscitan generalmente de forma solapada. En el caso de la acción "Algunos compañeros (as) no te dejan compartir con ellos, algún juego o conversación en el recreo", esta lleva implícita la exclusión de un niño o una niña de las actividades que se llevan a cabo durante el recreo. Estas son muy importantes para la población estudiantil, ya que acceden al logro de un eficiente desarrollo social y emocional. Es un espacio que resulta valioso, pues les permite relacionarse con otros: jugar, conversar, compartir la merienda, entre otras. Sin embargo, cuando no se deja participar al compañero o compañera de las actividades que se realizan en el recreo, existe un maltrato entre iguales que favorece el aislamiento y la soledad de niños y niñas víctimas de este tipo de violencia oculta. Los resultados que se obtuvieron permiten identificar que más del $50 \%$ 
de las y los encuestados, considera que en este tipo de situación sí se presenta un comportamiento violento. Al respecto, Arellano, Chirinos, López y Sánchez manifiestan que:

Toda situación de maltrato psíquico como el acoso, el maltrato a través de humillaciones, exclusión (incitando al grupo a que no estén con él o que no le hablen), amenazas, insultos, el descrédito y desprestigio, realizada de manera intensa o prolongada, provoca una serie de daños psíquicos, que incapacitan a la persona para defenderse. (2007, p.7)

El rechazo es también otro tipo de violencia psicológica que expone a la víctima delante de otros compañeros o compañeras, al aislamiento del grupo de pares, la soledad y la humillación de no ser aceptado ante otros. Arellano et al. (2007) indican que, cuando se rechaza, ignora o aísla a una persona de un grupo, o no se le permite participar de las actividades de su interés, se está ante un maltrato de tipo social. Asimismo, estas especialistas consideran que las consecuencias de este tipo de maltrato serán evidentes en las víctimas cuando estas presenten problemas para lograr relacionarse con otros de forma efectiva. Si se observa el cuadro 2, en el punto tres que indica que "Cuando algunos compañeros (as) se apartan de ti y no les gusta que juegues o salgas con ellos al recreo", un alto porcentaje de las y los niños de los tres grados encuestados califica este tipo de comportamiento como violento.

Respecto a la expresión "Cuando alguien te quita la merienda o alguno de los útiles escolares (lápiz, borrador, otros)", el cuadro permite identificar que un alto porcentaje de las y los encuestados manifiesta que sí existe violencia cuando el niño despoja a otro de su merienda. Este tipo de comportamientos violentos evidencian la existencia del ejercicio de poder de un niño sobre otro, donde la fuerza se impone sobre la voluntad de la persona que está siendo violentada. 


\begin{tabular}{|c|c|c|c|c|c|c|}
\hline \multicolumn{7}{|c|}{$\begin{array}{l}\text { Cuadro } 3 \\
\text { San Ramón: Opinión de las y los estudiantes de I Ciclo Enseñanza General Básica acerca de } \\
\text { las manifestaciones de violencia verbal que se podrían suscitar en los recreos, } 2012\end{array}$} \\
\hline \multirow[t]{2}{*}{ Manifestaciones de violencia verbal } & \multicolumn{3}{|c|}{ Hay violencia } & \multicolumn{3}{|c|}{ No hay violencia } \\
\hline & \multicolumn{3}{|c|}{ I grado II grado III grado } & \multicolumn{3}{|c|}{ I grado II grado III grado } \\
\hline $\begin{array}{l}\text { Algún compañero (a) le dijo a los } \\
\text { demás alguna cosa que te ofendió, } \\
\text { humilló o avergonzó. }\end{array}$ & $\begin{array}{l}91 \\
94,8\end{array}$ & $\begin{array}{l}90 \\
90,0\end{array}$ & $\begin{array}{c}75 \\
87,2\end{array}$ & $\begin{array}{c}5 \\
5,2\end{array}$ & $\begin{array}{l}10 \\
10,0\end{array}$ & $\begin{array}{l}11 \\
12,8\end{array}$ \\
\hline $\begin{array}{l}\text { Cuando los compañeros (as) te dicen } \\
\text { malas palabras y te hacen sentir mal. }\end{array}$ & $\begin{array}{l}89 \\
92,7\end{array}$ & $\begin{array}{c}91 \\
91,0\end{array}$ & $\begin{array}{c}80 \\
93,0\end{array}$ & $\begin{array}{l}7 \\
7,3\end{array}$ & $\begin{array}{l}9 \\
9,0\end{array}$ & $\begin{array}{c}6 \\
7,0\end{array}$ \\
\hline $\begin{array}{l}\text { Cuando los compañeros (as) te dicen } \\
\text { apodos. }\end{array}$ & $\begin{array}{l}80 \\
83,3\end{array}$ & $\begin{array}{c}79 \\
79,0\end{array}$ & $\begin{array}{c}66 \\
76,7\end{array}$ & $\begin{array}{c}16 \\
16,7\end{array}$ & $\begin{array}{c}21 \\
21,0\end{array}$ & $\begin{array}{l}20 \\
23,3\end{array}$ \\
\hline
\end{tabular}

Fuente: Creación propia a partir del cuestionario aplicado a las y los estudiantes de los grupos en estudio, 2012.

La violencia verbal es un tipo de maltrato que, a diferencia, por ejemplo, de la violencia física, no se percibe tan fácilmente, pero que, sin embargo, afecta en gran medida a la persona que recibe comentarios que la degradan, como insultos, gritos y frases que humillan, así como burlas y gestos humillantes. Generalmente, el victimario emite frases o palabras que pretenden provocar en la víctima sentimientos de rabia, humillación, vergüenza e impotencia. Al respecto, Barreras (2008) enuncia que hoy "el círculo socio-cultural nos incita a movernos en representaciones de competencia, de un lenguaje ofensivo y poco mediador. Este es el paradigma que se encuentra, en gran parte, en los medios de comunicación y en la sociedad" (p. 4). Al observar los resultados a las preguntas que tienen relación con las manifestaciones de violencia verbal (Cuadro 3), se logra identificar un alto porcentaje de niñas y niños que sí consideran que existe violencia si alguien les dice malas palabras, palabras que los ofenden o humillan, y apodos que los hacen sentirse mal. Sin embargo, es interesante observar que un $21 \%$ y $20 \%$ de estudiantes de II y III grado, respectivamente, enuncia que recibir apodos no es violencia. Pareciera que conforme avanza la edad del niño y la niña, algunos perciben que no existe violencia cuando se les dice palabras o expresiones que los hacen sentir mal. 


\section{Cuadro 4}

San Ramón: Opinión de las y los estudiantes de I Ciclo Enseñanza General Básica acerca de las manifestaciones de maltrato que se podrían suscitar en los recreos, 2012

\begin{tabular}{|c|c|c|c|c|c|c|}
\hline \multirow[t]{2}{*}{ Manifestaciones de maltrato } & \multicolumn{3}{|c|}{ Hay violencia } & \multicolumn{3}{|c|}{ No hay violencia } \\
\hline & \multicolumn{3}{|c|}{ I grado II grado III grado } & \multicolumn{3}{|c|}{ I grado II grado III grado } \\
\hline $\begin{array}{l}\text { Existen compañeros (as) que te } \\
\text { amenazan que te van a pegar. }\end{array}$ & $\begin{array}{l}92 \\
95,8\end{array}$ & $\begin{array}{l}88 \\
88,0\end{array}$ & $\begin{array}{c}83 \\
96,5\end{array}$ & $\begin{array}{l}4 \\
4,2\end{array}$ & $\begin{array}{l}12 \\
12,0\end{array}$ & $\begin{array}{l}3 \\
3,5\end{array}$ \\
\hline $\begin{array}{l}\text { Hay compañeros (as) que no te dejan } \\
\text { hablar o jugar con otros. }\end{array}$ & $\begin{array}{c}77 \\
80,2\end{array}$ & $\begin{array}{c}84 \\
84,0\end{array}$ & $\begin{array}{l}63 \\
73,3\end{array}$ & $\begin{array}{l}19 \\
19,8\end{array}$ & $\begin{array}{c}16 \\
16,0\end{array}$ & $\begin{array}{c}23 \\
26,7\end{array}$ \\
\hline $\begin{array}{l}\text { Cuando un compañero (a) te exige que } \\
\text { debes darle dinero, porque si no te va a } \\
\text { pegar. }\end{array}$ & $\begin{array}{c}90 \\
93,8\end{array}$ & $\begin{array}{l}96 \\
96,0\end{array}$ & $\begin{array}{c}81 \\
94,2\end{array}$ & $\begin{array}{c}6 \\
6,3\end{array}$ & $\begin{array}{c}4 \\
4,0\end{array}$ & $\begin{array}{c}5 \\
5,8\end{array}$ \\
\hline $\begin{array}{l}\text { Existen niños (as) que te prohíben decir } \\
\text { a la maestra, o darle quejas a la } \\
\text { maestra, cuando otros te molestan, } \\
\text { humillan, amenazan o pegan. }\end{array}$ & $\begin{array}{l}86 \\
89,6\end{array}$ & $\begin{array}{l}92 \\
92,0\end{array}$ & $\begin{array}{c}80 \\
93,0\end{array}$ & $\begin{array}{c}10 \\
10,4\end{array}$ & $\begin{array}{l}8 \\
8,0\end{array}$ & $\begin{array}{c}6 \\
7,0\end{array}$ \\
\hline $\begin{array}{l}\text { Cuando la maestra no escucha o no } \\
\text { presta atención a tus quejas, cuando } \\
\text { eres víctima de que otro niño o niña te } \\
\text { pegue, humille, diga malas palabras o } \\
\text { apodos y te amenace. }\end{array}$ & $\begin{array}{c}89 \\
92,7\end{array}$ & $\begin{array}{c}86 \\
86,0\end{array}$ & $\begin{array}{l}81 \\
94,2\end{array}$ & 7 & $\begin{array}{c}14 \\
14,0\end{array}$ & $\begin{array}{l}5 \\
5,8\end{array}$ \\
\hline
\end{tabular}

Fuente: Creación propia a partir del cuestionario aplicado a las y los estudiantes de los grupos en estudio, 2012.

Existe un tipo de violencia que resulta muy difícil de percibir. Es aquella que podemos denominar como oculta, porque es un fenómeno que las víctimas sufren frecuentemente y que se desarrolla bajo condiciones que podrían resultar ser quizá más dañinas que aquellos comportamientos violentos que se suscitan a través del golpe, el empujón, la zancadilla, el pellizco. El maltrato que sufren diariamente niños y jóvenes en los ambientes escolares, en la mayoría de los casos se lleva a cabo de forma solapada, oculta, y estos son víctimas del acoso, rechazo, indiferencia, amenazas, hostilidad, entre otros.

El cuadro 4 muestra información relacionada con el maltrato escolar, donde el niño o la niña recibe amenazas de otro u otra compañera o no es escuchado por parte del adulto, en este caso en particular, por la o el docente. Obsérvese que un alto porcentaje de las y los niños encuestados identifica la existencia de violencia en las acciones ligadas a comportamientos violentos que conllevan la amenaza de unos niños sobre otros. Asimismo, un alto porcentaje de estos estudiantes considera que, cuando la docente no presta atención a aquellas quejas que tienen que ver con los procesos de victimización de actos violentos, están ante una situación de violencia. Para Buitrago, Cabrera, Guevara y Sánchez (2009), el 
docente juega un papel fundamental en la intervención de situaciones de intimidación entre estudiantes. Es necesario, entonces, que el docente se mantenga abierto y atento ante los comportamientos violentos que se susciten entre sus estudiantes $y$, tal y como lo indican estas autoras

Es conveniente que el maestro inspire confianza para que se establezca una comunicación fluida con fuerte espíritu crítico, de tal manera que una interacción no llegue como una imposición sino como algo agradablemente acertado; estar disponible, quiere decir que se facilite a los estudiantes acudir en el momento preciso. (2009, p.76)

Las y los estudiantes, cuando son víctimas de manifestaciones de violencia, necesitan hallar a un adulto, en este caso un o una docente que promueva un ambiente de seguridad, donde sientan que van a encontrar disposición por parte del adulto a escuchar sus quejas y que busque los mecanismos pertinentes para lograr una intervención que fomente el cese y aborde de forma pertinente la situación de maltrato.

\section{Cuadro 5}

San Ramón: Opinión de las y los estudiantes de I Ciclo escuela en estudio acerca de las manifestaciones de violencia sexual que se podrían suscitar en los recreos, 2012

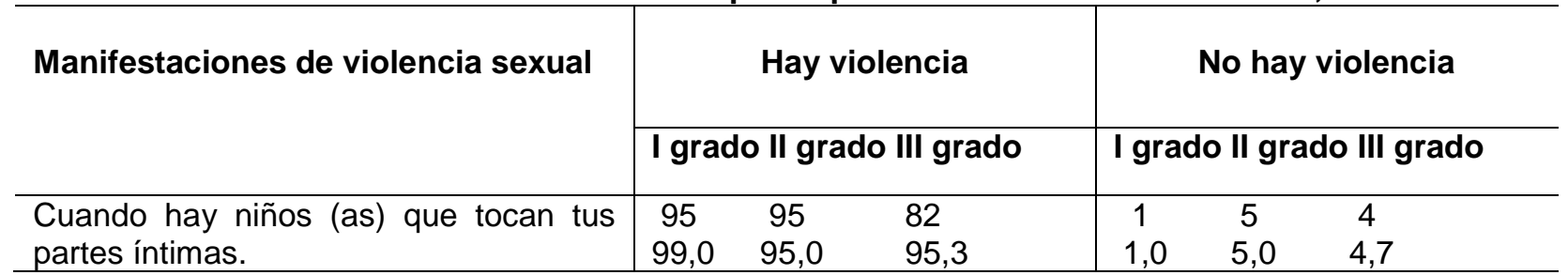

Fuente: Creación propia a partir del cuestionario aplicado a las y los estudiantes de los grupos en estudio, 2012.

Para Capriles (2008) el acoso sexual es "una manifestación oculta que apunta fundamentalmente hacia el maltrato, la agresión o el acoso de carácter sexual" (p. 180). Asimismo, el autor indica que este tipo de violencia no es exclusiva del adulto, sino que también participan los iguales en la escuela. La información que se presenta en el cuadro 5 permite reconocer que un alto porcentaje de las y los estudiantes considera que sí existe violencia cuando alguien toca sus partes íntimas. 
A continuación se presentan una serie de ítemes que complementan los anteriores. En estos se les mostraron a los estudiantes acciones donde no existen comportamientos violentos.

\section{Cuadro 6}

San Ramón: Opinión de los estudiantes de I ciclo Enseñanza General Básica acerca de las manifestaciones de violencia que se podrían suscitar en los recreos, 2012

\begin{tabular}{|c|c|c|c|c|c|c|}
\hline \multirow{3}{*}{$\begin{array}{l}\text { Manifestaciones de maltrato } \\
\begin{array}{l}\text { Cuando sales a jugar con los } \\
\text { compañeros. }\end{array}\end{array}$} & \multicolumn{3}{|c|}{ Hay violencia } & \multicolumn{3}{|c|}{ No hay violencia } \\
\hline & \multicolumn{3}{|c|}{ I grado II grado III grado } & \multicolumn{3}{|c|}{ I grado II grado III grado } \\
\hline & $\begin{array}{c}1 \\
1,0 \\
\end{array}$ & $\begin{array}{c}0 \\
0,0\end{array}$ & $\begin{array}{c}1 \\
1,2\end{array}$ & $\begin{array}{c}95 \\
99,0\end{array}$ & $\begin{array}{l}100 \\
100,0\end{array}$ & $\begin{array}{l}85 \\
98,8\end{array}$ \\
\hline $\begin{array}{l}\text { Cuando compartes la merienda con tus } \\
\text { compañeros. }\end{array}$ & $\begin{array}{c}3 \\
3,1\end{array}$ & $\begin{array}{l}2 \\
2,0\end{array}$ & $\begin{array}{l}2 \\
2,3\end{array}$ & $\begin{array}{c}93 \\
96,9\end{array}$ & $\begin{array}{c}98 \\
98,0\end{array}$ & $\begin{array}{l}84 \\
97,7\end{array}$ \\
\hline $\begin{array}{l}\text { Si asistes al gimnasio a jugar con los } \\
\text { compañeros. }\end{array}$ & $\begin{array}{c}1 \\
1,0 \\
\end{array}$ & $\begin{array}{c}2 \\
2,0 \\
\end{array}$ & $\begin{array}{c}1 \\
1,2\end{array}$ & $\begin{array}{l}95 \\
99,0\end{array}$ & $\begin{array}{c}98 \\
98,0\end{array}$ & $\begin{array}{c}85 \\
98,8\end{array}$ \\
\hline $\begin{array}{l}\text { Cuando vas a la soda con un } \\
\text { compañero } \\
\text { alimento a a comparar algún } \\
\text { llim }\end{array}$ & $\begin{array}{c}1 \\
1,0\end{array}$ & $\begin{array}{c}3 \\
3,0\end{array}$ & $\begin{array}{c}0 \\
0,0\end{array}$ & $\begin{array}{c}95 \\
99,0\end{array}$ & $\begin{array}{l}97 \\
97,0\end{array}$ & $\begin{array}{l}86 \\
100,0\end{array}$ \\
\hline $\begin{array}{l}\text { Cuando compartes juegos con los } \\
\text { compañeros como brincar la cuerda, } \\
\text { elástico, bola, correr. }\end{array}$ & $\begin{array}{c}4 \\
4,2\end{array}$ & $\begin{array}{l}2 \\
2,0\end{array}$ & $\begin{array}{c}5 \\
5,8\end{array}$ & $\begin{array}{c}92 \\
95,8\end{array}$ & $\begin{array}{c}98 \\
98,0\end{array}$ & $\begin{array}{l}81 \\
94,2\end{array}$ \\
\hline $\begin{array}{l}\text { Cuando gritas, caminas o corres con los } \\
\text { compañeros (as) por los pasillos. }\end{array}$ & $\begin{array}{l}14 \\
14,6\end{array}$ & $\begin{array}{l}11 \\
11,0\end{array}$ & $\begin{array}{l}18 \\
20,9\end{array}$ & $\begin{array}{l}82 \\
85,4\end{array}$ & $\begin{array}{c}89 \\
89,0\end{array}$ & $\begin{array}{l}68 \\
79,1\end{array}$ \\
\hline
\end{tabular}

Fuente: Creación propia a partir del cuestionario aplicado a las y los estudiantes de los grupos en estudio, 2012.

El cuadro 6 permite observar seis ítemes que se les presentaron a los estudiantes y que están relacionados con la actividad lúdica y de interacción personal que desarrollan durante los recreos. El objetivo de estos ítemes fue tratar de identificar si la población estudiantil de I, II y III grado de la escuela en estudio, lograba reconocer si en estas acciones se suscitaban comportamientos violentos. Como se puede observar, un alto porcentaje de estudiantes consideran que en estas actividades no existe violencia. Esto permite observar que estos estudiantes logran percibir cuando existen o no comportamientos violentos entre los que comparten actividades lúdicas durante el recreo.

Por otra parte, el cuadro 7 también presenta una serie de ítemes que hacen referencia a algunas actividades vinculadas a relaciones interpersonales que niños y niñas podrían compartir durante los recreos. Obsérvese que, de las actividades que se les presentó, únicamente dos de estas logran ser percibidas por los estudiantes como violentas. 
Específicamente, se considera importante referirse al ítem que hace referencia a "Cuando buscas a la maestra para decirle que algún niño (a) está peleando con otro (a)", el cual es percibido por un $51 \%$ de los niños y niñas de primer grado como violencia. Al estar la investigadora en contacto directo con la población en estudio mientras estos contestaban la pregunta, esta pudo comprender la razón de los resultados, pues algunos de los niños comentaban que sí había violencia porque los "chiquitos estaban peleando".

Sin embargo, es importante resaltar que un $47 \%$ de los compañeros de I grado y un $78 \%$ de II y III grado, identifica que en esta actividad no existe ningún comportamiento violento.

\section{Cuadro 7}

San Ramón: Opinión de los estudiantes de I ciclo Enseñanza General Básica acerca de las manifestaciones de violencia que se podrían suscitar en los recreos, 2012

\section{Manifestaciones de maltrato}

Hay violencia

No hay violencia

\begin{tabular}{|c|c|c|c|c|c|c|}
\hline \multirow[b]{2}{*}{$\begin{array}{l}\text { Cuando ayudas a un compañero (a) } \\
\text { que ha tenido un accidente en el recreo. }\end{array}$} & \multicolumn{3}{|c|}{ I grado II grado III grado } & \multicolumn{3}{|c|}{ I grado II grado III grado } \\
\hline & $\begin{array}{c}1 \\
1,0\end{array}$ & $\begin{array}{c}3 \\
3,0\end{array}$ & $\begin{array}{c}1 \\
1,2\end{array}$ & $\begin{array}{c}95 \\
99,0\end{array}$ & $\begin{array}{l}97 \\
97,0\end{array}$ & $\begin{array}{c}85 \\
98,8\end{array}$ \\
\hline $\begin{array}{l}\text { Cuando felicitas a un compañero (a) por } \\
\text { ganar en alguno de los juegos. }\end{array}$ & $\begin{array}{l}2 \\
2,1\end{array}$ & $\begin{array}{l}0 \\
0,0\end{array}$ & $\begin{array}{c}1 \\
1,2\end{array}$ & $\begin{array}{c}94 \\
97,9\end{array}$ & $\begin{array}{c}100 \\
100,0\end{array}$ & $\begin{array}{c}85 \\
98,8\end{array}$ \\
\hline $\begin{array}{l}\text { Cuando acompañas a un compañero } \\
\text { (a) que está solo, porque nadie quiere } \\
\text { jugar con él. }\end{array}$ & $\begin{array}{c}10 \\
10,4\end{array}$ & $\begin{array}{c}11 \\
11,0\end{array}$ & $\begin{array}{c}10 \\
11,6\end{array}$ & $\begin{array}{c}86 \\
89,6\end{array}$ & $\begin{array}{l}89 \\
89,0\end{array}$ & $\begin{array}{c}76 \\
88,4\end{array}$ \\
\hline Al conversar con los compañeros (as). & $\begin{array}{c}2 \\
2,1\end{array}$ & $\begin{array}{c}0 \\
0,0\end{array}$ & $\begin{array}{c}3 \\
3,5\end{array}$ & $\begin{array}{c}94 \\
97,9\end{array}$ & $\begin{array}{c}100 \\
100,0\end{array}$ & $\begin{array}{c}83 \\
96,5\end{array}$ \\
\hline $\begin{array}{l}\text { Al compartir tu dinero con un } \\
\text { compañero al que se le perdió el suyo. }\end{array}$ & $\begin{array}{l}7 \\
7,3\end{array}$ & $\begin{array}{r}4 \\
4,0\end{array}$ & $\begin{array}{c}1 \\
1,2\end{array}$ & $\begin{array}{c}89 \\
92,7\end{array}$ & $\begin{array}{c}96 \\
96,0\end{array}$ & $\begin{array}{c}85 \\
98,8\end{array}$ \\
\hline $\begin{array}{l}\text { Cuando le dices a un compañero (a) } \\
\text { palabras que lo hacen sentir bien, feliz. }\end{array}$ & $\begin{array}{c}8 \\
8,3\end{array}$ & $\begin{array}{r}5 \\
5,0\end{array}$ & $\begin{array}{c}3 \\
3,5\end{array}$ & $\begin{array}{l}88 \\
91,7\end{array}$ & $\begin{array}{r}95 \\
95,0\end{array}$ & $\begin{array}{c}83 \\
96,5\end{array}$ \\
\hline $\begin{array}{l}\text { Cuando ayudas a un compañero (a) } \\
\text { que es amenazado por otro. }\end{array}$ & $\begin{array}{c}20 \\
20,8\end{array}$ & $\begin{array}{c}14 \\
14,0\end{array}$ & $\begin{array}{l}8 \\
9,3\end{array}$ & \begin{tabular}{|l|}
76 \\
79,2
\end{tabular} & $\begin{array}{c}86 \\
86,0\end{array}$ & $\begin{array}{c}78 \\
90,7\end{array}$ \\
\hline $\begin{array}{l}\text { Cuando buscas a la maestra para } \\
\text { decirle que algún niño (a) está peleando } \\
\text { con otro (a). }\end{array}$ & $\begin{array}{c}49 \\
51,0\end{array}$ & $\begin{array}{r}22 \\
22,0\end{array}$ & $\begin{array}{c}18 \\
20,9\end{array}$ & $\begin{array}{c}47 \\
49,0\end{array}$ & $\begin{array}{r}78 \\
78,0\end{array}$ & $\begin{array}{r}68 \\
79,1\end{array}$ \\
\hline
\end{tabular}

Fuente: Creación propia a partir del cuestionario aplicado a las y los estudiantes de los grupos en estudio, 2012.

Como parte del análisis estadístico que se llevó a cabo con la información suministrada a partir de los 30 ítemes antes citados, se logró identificar el nivel de percepción de los comportamientos violentos que poseen las y los estudiantes. Antes de brindar estos datos, 
corresponde explicar el tratamiento que se les dio. En primer lugar, es preciso indicar que a cada uno de los 30 ítemes, se procedió a asignársele 2 puntos, si el estudiante lograba detectar de forma correcta en cada ítem la presencia de un comportamiento violento, y 0 puntos a aquellas respuestas donde los estudiantes no lograran identificar los comportamientos violentos en la redacción de las acciones que se presentaron.

El índice de comportamientos violentos se calculó tomando en cuenta los 30 ítemes, los cuales se transformaron en una escala de 0 a 10, donde 0 es el caso más extremo y, por tanto, ninguna de las acciones que se presentan son violentas. El 10, por su parte, indica que la persona logra percibir que las acciones que se le presentan son comportamientos violentos. Los resultados permiten identificar que la media del promedio de conocimiento acerca de los comportamientos violentos para los estudiantes de I, II y III grado alcanza el 9,0. Por tanto, se puede señalar que la población estudiantil del I Ciclo de la Enseñanza General Básica de la escuela en estudio, consigue identificar las conductas violentas presentes en aquellos ítemes que así lo muestran, lo que permite indicar la existencia de un alto índice de conocimiento de los comportamientos violentos.

\section{Reflexiones finales}

En cuanto a la percepción que tienen las y los estudiantes de I Ciclo de la Enseñanza General Básica de una escuela de la Dirección Regional de Educación de Occidente acerca de la violencia escolar, este estudio permitió llegar a las siguientes conclusiones:

- Un alto porcentaje de las y los estudiantes de I, II y III grado de la escuela en estudio, identifica de forma categórica los diferentes tipos de violencia que se podrían suscitar en la escuela: física, psicológica, verbal y sexual.

- Respecto a las acciones que se presentaron en las ilustraciones y en las aseveraciones de los ítemes que hacían referencia al desarrollo de la violencia física en el cuestionario, un alto porcentaje de niños y niñas de los tres grados reconoció la existencia de acciones violentas de tipo físico. Con porcentajes mayores del $90 \%$ de respuestas afirmativas, las y los estudiantes identifican que, cuando alguien los empuja, les pega zancadillas y los golpea, están siendo víctimas de violencia física.

- En cuanto a la violencia de tipo psicológica, las y los estudiantes en su mayoría opinan que en las acciones que se presentan en los ítemes correspondientes, sí existe violencia. Este tipo de violencia no resulta ser tan evidente como la física, se produce 
de forma sutil y es difícil de detectar para quienes la ejercen y quienes la padecen. Sin embargo, es importante destacar que la gran mayoría de los niños y niñas sujetos del estudio, lograron detectar que en las acciones que se les presentaron y que ilustraban relaciones sociales cotidianas, sí existían comportamientos violentos.

- Al cuestionar respecto a la violencia verbal, un alto porcentaje de las y los estudiantes manifiesta que sí existe violencia cuando alguien divulga cosas que ofenden, humillan o avergüenzan, dicen malas palabras o apodos. Este tipo de violencia presente en las ilustraciones y acciones, fue percibida por niños y niñas como comportamientos violentos que ofenden, humillan y lastiman al otro.

- Respecto a las manifestaciones de intimidación entre estudiantes, como amenazas, exigencia de dinero a cambio de no maltratar, no permitir que alguien hable o juegue con otros, así como cuando el personal docente no escucha las quejas, son consideradas por un alto porcentaje de estudiantes como violentas. Este tipo de comportamientos son generados a partir de la violencia verbal y la psicológica, surgen como comportamientos que no son perceptibles a simple vista. Los estudiantes lograron identificar eficientemente las manifestaciones de la violencia intimidatoria: exclusión adrede del otro, el rechazo, el aislamiento y la intimidación.

- Un alto porcentaje de estudiantes reconoce la existencia de violencia sexual. Este tipo de violencia es "invisible" y sólo se concreta a través del hostigamiento y tocamientos. En el caso de los estudiantes del estudio, más del 90\% logró identificar que cuando otros tocan sus partes íntimas, se está cometiendo un acto de violencia.

- El análisis estadístico empleado en el estudio reveló que la población estudiantil del I Ciclo de la Enseñanza General Básica de la escuela en estudio, consigue identificar las conductas violentas presentes en aquellos ítemes que así lo muestran, lo que permite indicar la existencia de un alto índice de conocimiento de los comportamientos violentos por parte de las y los estudiantes.

\section{Referencias}

Abad, Susana. (2002). Acerca de las conductas violentas en el aula. Cuando la asimetría lleva a la humillación. Ensayos y experiencias, 44, 30-45. 
Álvarez, Luis, Álvarez, David, González, Paloma, Núñez, José Carlos y González, Julio Antonio. (2006). Evaluación de los comportamientos violentos en los centros educativos. Psicothema, 18(4), 686-695.

Arellano, Norka, Chirinos Yennis, López Zugeidy y Sánchez Lissett. (2007). Los tipos de maltrato entre iguales. Recuperado de http://www.quadernsdigitals.net/index.php?accionMenu=hemeroteca. DescargaArticulol U.descarga\&tipo=PDF\&articulo $\mathrm{id}=10404$

Arias, Leonel. (2009). Una mirada al fenómeno de la violencia escolar en Costa Rica. Revista Electrónica Educare, 13(1). 41-51. Recuperado de: http://www.revistas.una.ac.cr/index.php/EDUCARE/article/view/1475/0

Artavia, Jenny. (2012). Comportamientos violentos en los juegos e interacciones sociales que se suscitan entre las y los estudiantes de I Ciclo de la Enseñanza General Básica durante el desarrollo de los recreos, en una escuela del circuito 01 de la Dirección Regional de Educación de Occidente (Informe parcial de investigación, N 724- B1901). San José, C.R.: INIE, Universidad de Costa Rica.

Barreras, Luis. (2008). La comunicación y la violencia escolar: jóvenes, lenguaje y violencia. Ponencia presentada al $10^{\circ}$ CONGRESO REDCOM "Conectados, Hipersegmentados y Desinformados en la Era de la Globalización”. Universidad Católica de Salta, Argentina. Recuperado de http://observatorioperu.com/lecturas\%202010/abril\%202010/abril2/LA\%20COMUNICA CION\%20Y\%20LA\%20VIOLENCIA\%20ESCOLAR\%20JOVENES,\%20LENGUAJE\%20 Y\%20VIOLENCIA..pdf

Benítez, Juan Luis y Justicia, Fernando. (2006). El maltrato entre iguales: descripción análisis del fenómeno. Revista electrónica de Investigación Psicoeducativa, 4(9), 151-170. Recuperado de http://www.investigacionpsicopedagogica.org/revista/new/ContadorArticulo.php?114

Buitrago, María del Pilar, Cabrera, Karol, Guevara, Mónica y Sánchez, Niny. (2009). Intimidación escolar, escuela y familia: una triada al borde de una perspectiva más humana y social. Aletheia. Revista de desarrollo humano, educativo y social contemporáneo, 1(1), 67-79. http://aletheia.cinde.org.co/index.php/ALETHEIA/article/view/6

Cabrera, Elsa Piedad. (2005). Palabras que dejan huella: violencia en la escuela a través del discurso. Revista Iberoamericana de Educación, (37), 49-54.

Capriles, Clarisa. (2008). Modernidad, educación y violencia: rasgos distintivos de la escuela contemporánea. Ide@s CONCYTEG, 3(36). Recuperado de http://www.concyteg.gob.mx/ideasConcyteg/Archivos/36122008 MODERNIDAD EDU CACION VIOLENCIA.pdf

Cerezo, Fuensanta. (2002). Conductas agresivas en la edad escolar. Aproximación teórica y metodológica. Propuestas de intervención. Madrid: Pirámide. 
Fundación Piquer. (2010). Violencia y acoso escolar. Recuperado de: www.observatorioperu.com/lecturas2010/julio2010/VIOLENCIAYACOSOESCOLARPIQUER.pdf

Hernández, Mario. (2008). La violencia en las escuelas: un problema actual a solucionar por la Educación, la Ciencia, la Tecnología y la Sociedad. Revista Iberoamericana de Educación, (46), 1-12. Recuperado de http://www.rieoei.org/deloslectores/2038Nodarse.pdf

Martínez-Otero, Valentín. (2005). Conflictividad escolar y fomento de la convivencia. Revista Iberoamericana de Educación, (38), 89-107.

Palomero, José Emilio y Fernández, María Rosario. (2001). La violencia escolar: un punto de vista global. Revista Interuniversitaria de Formación del profesorado, (41), 19-38. Recuperado de www.aufop.com/aufop/uploaded files/articulos/1247352775.pdf 